\title{
Impact of model resolution on numerical weather prediction
}

\author{
Z.SAHLAOUI ${ }^{1,2}$, S. MORDANE ${ }^{2}$
}

1. CNRMSI, Direction de la Météorologie Nationale B.P. 8106 Casablanca - Maroc sahlaoui_zahra@yahoo.fr

2. LPPPC, Faculté des Sciences Ben M’sik, Univetsité Hassan II Casablanca - Maroc mordanesoumia@yahoo.fr

\begin{abstract}
Several model configurations are used in Morocco for numerical weather prediction (NWP). The aim of this work is to verify the impact of resolution on the quality of models forecast, particularly the precipitation field. Three model configurations are tested with $7.5 \mathrm{~km}, 5 \mathrm{~km}$ and $2.5 \mathrm{~km}$ resolution. A rainy event over the North-East of Morocco is studied. The impact on models performances is assessed through the comparison of precipitation forecasts with the adjusted quantitative precipitation estimate from weather radar. The results show that the model with $2.5 \mathrm{~km}$ resolution gives the best quality precipitation forecast in term of both intensity and localisation.
\end{abstract}

\section{Keywords: Numerical models, weather prediction, resolution, precipitation forecast.}

\section{Introduction}

The weather phenomena have a growing impact on human safety and economic activities. Thus, producing good quality forecast is highly useful especially in case of precipitation events. The modelling of the atmosphere is an essential tool for producing weather forecast. A large variety of numerical weather models was developed to simulated atmospheric behaviour in global, regional and local scale. The model forecast accuracy mainly depends on the numerical simulation of the atmospheric processes and on the model resolution.

In the present work, the ability of the model to improve precipitation forecast and the add value of finer resolution will be tested through numerical experiments using three limited area models (LAM) configurations. A case study over the North-East of Morocco will be carried. The impact on models performances will be assessed through the comparison of precipitation forecasts with the adjusted quantitative precipitation estimate from radar data.

\section{Modeling the atmosphere with ALADIN, ALARO and AROME}

The Moroccan Meteorological Administration runs operationally three NWP models [1] that are ALADIN ${ }^{1}$, $\mathrm{AROME}^{2}$ and $\mathrm{ALARO}^{3}$ (table 1). ALADIN was the first LAM version used in Morocco for regional forecast at a resolution of about $10 \mathrm{~km}$. Later, the two models AROME and ALARO were introduced to satisfy the needs of mesoscale applications. The common code is based on equations built from fundamental conservation laws governing the physical behaviour of the atmosphere. The three models solve the fully compressible Euler Equation System. Additional approximations are used [2]: the atmosphere is considered as a perfect gas, the atmosphere thickness is neglected regarding the Earth radius and the vertical component of Coriolis acceleration is neglected too. The isolate system hypothesis is also applied, thus the atmosphere is considered adiabatic and without friction. Equations 1 to 6 are for the prognostic model variables (three wind components $V(\mathrm{u}, \mathrm{v}), w$, temperature $T$, pressure $P$ and surface hydrostatic pressure $\pi_{s}$ ). The last two equations are for the diagnostic variables (geopotential $\phi$ and generalized vertical velocity $\dot{s}$ ):

1. Continuity:
$\frac{\partial}{\partial t}\left(\frac{\partial \pi}{\partial s}\right)=-\nabla \cdot\left(\frac{\partial \pi}{\partial s} V\right)-\frac{\partial}{\partial s}\left(\frac{\partial \pi}{\partial s} \dot{s}\right)$ $s$ is the generalized vertical coordinate [3].

2. Horizontal motion 
$\frac{\partial V}{\partial t}=-k \times(\zeta+f) V-\dot{s} \frac{\partial \pi}{\partial s} \frac{\partial V}{\partial \pi}-\nabla \mathrm{K}-\left(\frac{\partial \mathrm{p}}{\partial s}\left(\frac{\partial \pi}{\partial s}\right)^{-1} \nabla \Phi+\frac{R T}{p} \nabla \ln p\right)$

$R$ is perfect gas constant, $f$ is Coriolis parameter,

$\zeta=k \cdot(\nabla \times f) V$ and $K=\|V\|^{2} / 2$

3. Hydrostatic

$\gamma \frac{\partial w}{\partial t}=-g\left[1-\frac{\partial P}{\partial s}\left(\frac{\partial \pi}{\partial s}\right)^{-1}\right]$

The $\gamma$ factor is used to distinct the hydrostatic $(\gamma=0)$ and non-hydrostatic $(\gamma=1)$ cases.

4. Temperature

$\frac{\partial T}{\partial t}=-V \cdot \nabla T-\dot{s} \frac{\partial \pi}{\partial s} \frac{\partial T}{\partial \pi}+\frac{R T}{C_{p}} \frac{d \ln P}{d t}$

5. Pressure

$\frac{\partial \ln P}{\partial t}=-V \cdot \nabla \ln P-\dot{s} \frac{\partial \pi}{\partial s} \frac{\partial \ln P}{\partial \pi}$

$-\frac{C_{p}}{C_{v}}\left[\nabla \cdot \mathrm{V}+\frac{P}{R T}\left(\frac{\partial \pi}{\partial s}\right)^{-1}\left(\frac{\partial V}{\partial s}\right) \cdot \nabla \Phi-g \frac{P}{R T}\left(\frac{\partial \pi}{\partial s}\right)^{-1} \frac{\partial w}{\partial s}\right]$

$C_{p}$ and $C_{v}$ are latent capacity at constant pressure,

volume respectively.

6. Surface hydrostatic pressure

$\frac{\partial \pi_{s}}{\partial t}=-\int_{s_{t}}^{s_{b}} \nabla \cdot\left(\frac{\partial \pi}{\partial s} V\right) d s$

7. Generalized vertical velocity

$\left(\dot{s} \frac{\partial \pi}{\partial s}\right)=-\int_{s_{t}}^{s} \nabla \cdot\left(\frac{\partial \pi}{\partial \dot{s}} V\right) d \dot{s}+\frac{\partial \pi}{\partial \pi_{s}} \int_{s_{t}}^{s_{b}} \nabla \cdot\left(\frac{\partial \pi}{\partial s} V\right) d s$

8. Geopotential

$\Phi=\Phi_{s}-\int_{s_{b}}^{s} \frac{R T}{p} \frac{\partial \pi}{\partial \dot{s}} d \dot{s}$

The numerical resolution of the system uses bi-Fourier spectral decomposition in both horizontal directions. In the vertical, the finite difference (FD) method is applied at model levels. Centred FD algorithms are used to compute vertical derivatives where vertical integrals are computed following mid-point rule. The time integration uses semi-implicit scheme [4].

Both ALADIN and AROME have hydrostatic dynamics while the AROME model has non-hydrostatic dynamics [5].

\begin{tabular}{|l|l|l|}
\hline Model & $\begin{array}{l}\text { Horizontal } \\
\text { resolution }\end{array}$ & Dynamics \\
\hline ALADIN & $7.5 \mathrm{~km}$ & Hydrostatic \\
\hline ALARO & $5 \mathrm{~km}$ & Hydrostatic \\
\hline AROME & $2.5 \mathrm{~km}$ & Non-Hydrostatic \\
\hline
\end{tabular}

Table 1: characteristics of NWP models in Morocco

\section{Impact of resolution and initial conditions on precipitation forecast}

\subsection{Case overview}

During the $19^{\text {th }}$ January 2018, a meteorological perturbation affected the North-East of Morocco (figure 1.a). The situation was characterised by a surface depression centred on the East of Morocco and by geopotential minimum associated to cold air $\left(-25^{\circ} \mathrm{C}\right)$ at $500 \mathrm{hPa}$ (figure 1.b). This configuration produced a cyclonic circulation bringing instable and humid air from the Mediterranean Sea (figure 1.c). Moderate precipitations reaching $30 \mathrm{~mm}$ in 24 hours occurs over the North-East regions in Morocco during this situation.
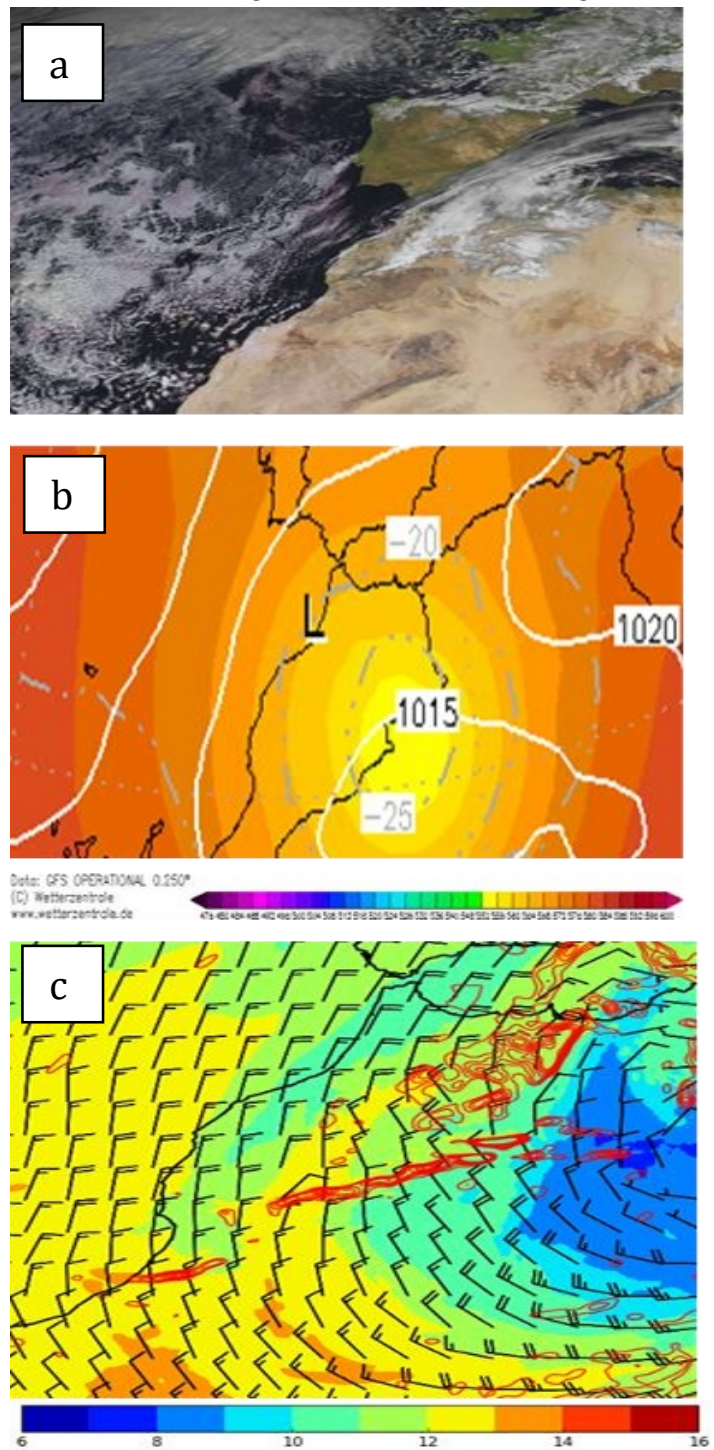

Figure 1: $19^{\text {th }}$ January 2018 meteorological situation: a) $\mathrm{MSG}^{4}$ image at 12UTC, b) Surface pressure, geopotential and temperature at $500 \mathrm{hPa}$ at 00UTC produced by GFS model, c) $600 \mathrm{hPa}$ wind, vertical velocity and temperature at $12 \mathrm{UTC}$.

\subsection{Forecast quality assessment}

To evaluate the performance of each model, especially the accuracy of the precipitation forecast, three

${ }^{4}$ Meteosat Second Generation 
experiments were run for ALADIN, ALARO and AROME.

The forecast of cumulative precipitation amount was compared to the quantitative precipitation estimate produced by the Debdou radar and adjusted by the surrounding rain gauges [6].

Figure 2 shows the precipitation amount from the $19^{\text {th }}$ at 06UTC to the $20^{\text {th }}$ at 06UTC January 2018, produced by the three experiments and observed by Debdou radar. Two precipitations cells of more that $30 \mathrm{~mm}$ are identified on the radar estimate (figure 2.a). The larger one (A) is located over Nador city and the surrounding area when the second one (B) is centred over Debdou city. The ALADIN experiment reproduces these two cells (figure 2.b) but the cell $\mathrm{A}$ is moved eastward. The ALARO experiment produces the same configuration as ALADIN with higher precipitation intensity for cell A and lower for cell B (figure 2.c). Concerning the AROME model, vertical motion of wet and warm air mass was detected over the study area (not shown). North flux at upper air brings humidity over the Moroccan Mediterranean coast, especially near Nador city. This atmospheric behaviour enhanced the precipitation forecast. Indeed, AROME produces the two cells with correct location and intensity (figure 2.d). Thus it can be deduced that this experiment is the best matching with the radar field.

\section{Conclusion}

This work was an opportunity to access the accuracy of precipitation forecast for many model resolution: ALADIN-7.5km versus ALARO-5km versus AROME$2.5 \mathrm{~km}$. Three experiments were run and the comparison with radar precipitation estimate allowed establishing relevant behaviour of each experiment. ALADIN and ALARO successfully reproduce the intensity of the main precipitation cells but the localisation was missed. The AROME model substantially improved the precipitation forecast for both intensity and localisation.

\section{References}

[1] P. Termonia et al, The ALADIN System and its canonical model configurations AROME CY41T1 and ALARO CY4OT1, Geosci. Model Dev. (2018), 11, 257-281.

[2] J. Coiffier, Les bases de la prévision numérique du temps, Société Météorologique de France, 2009.

[3] R. Laprise, The Euler equations of motion with hydrostatic pressure as an independent variable, Mon. Wea. Rev.(1992), 120, 197-207.

[4] A. J. Simmons et al, Stability of the semiimplicit method of time integration, Mon. Weather Rev. (1978),405-412.
[5] Y. Seity et al, The AROME-France ConvectiveScale Operational Model. Mon. Wea. Rev (2011)., 139, 976-991.

[6] Z. Sahlaoui, Amélioration de l'estimation de pluie par le radar, 1st International Congress MORGEO, Casablanca, Morocco, 2017.
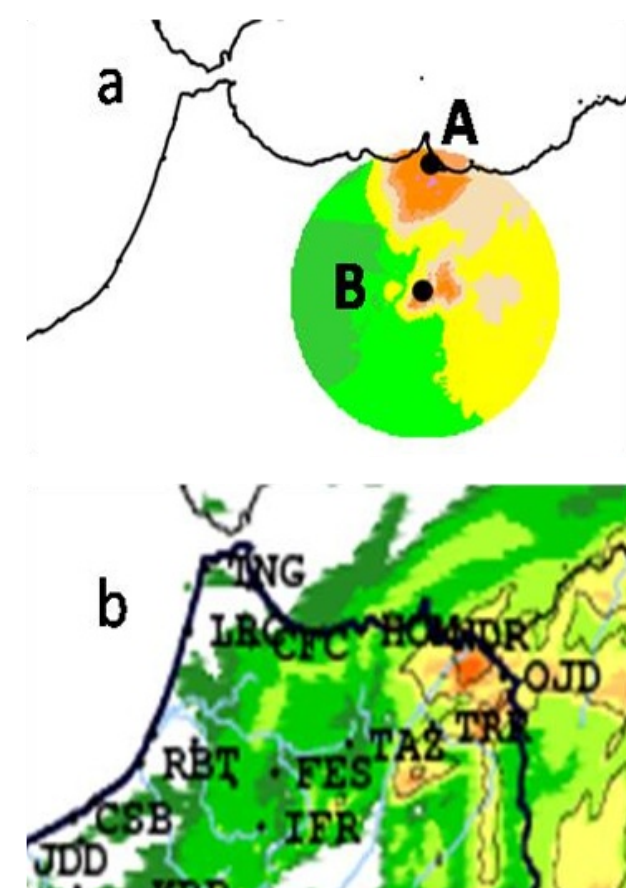

80.0

60.0
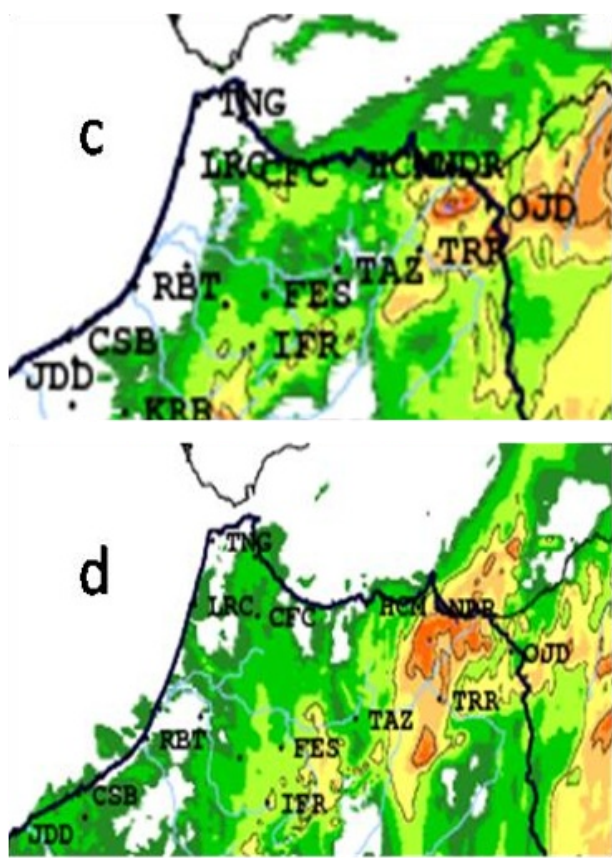

Figure 2: Precipitation amount from $19^{\text {th }}$ at $06 \mathrm{UTC}$ to $20^{\text {th }}$ at 06UTC January 2018, produced by Debdou radar (a), ALADIN (b), ALARO (c) and AROME (d). 